\title{
Training Pathways in Endodontics - Operative Dentistry or Conservative Dentistry Across the Globe and a Discourse on its Training in Pakistan
}

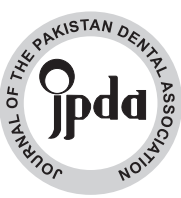

\author{
Farhan Raza Khan
}

BDS, MS, MCPS, FCPS

The objective of the paper is to present a mini review of training pathways in the specialty of Endodontics-Operative Dentistry or Conservative Dentistry across the globe and draw the attention of the readers towards the two models of training that exist in this specialty in Pakistan.

KEYWORDS: dental education; Operative Dentistry; Endodontics

HOW TO CITE: Khan FR. Training pathways in endodontics - operative dentistry or conservative dentistry across the globe and a discourse on its training in Pakistan. J Pak Dent Assoc 2021;30(3):144-146.

DOI: https://doi.org/10.25301/JPDA.303.144

Received: 27 December 2020, Accepted: 04 March 2021

$\mathrm{T}$ he objective of the paper is to present a mini review of training pathways in the specialty of EndodonticsOperative Dentistry or Conservative Dentistry across the globe and draw the attention of the readers towards the two models of training that exist in this specialty in Pakistan.

In USA and Canada, operative dentistry is not a subject specialty but endodontics is. ${ }^{1}$ The endodontics training in the dental institutions of North America spans from three to four years. The residency program has to be fully accredited by the Commission on Dental Accreditation (CODA). The training is quite focused on diagnosis and management of pulp and peri-radicular diseases, however, programs do vary with respect their time allocated for surgical endodontics and emphasis on research. The exit level qualification in USA is known as the Diplomat of the American Board in Endodontics whereas in Canada it's the Fellowship in Endodontics at the Royal College of Dentists.

In UK and Ireland, dentists are eligible to start their post graduate endodontics training once they have already done their post-BDS, two years of general professional training. The residency training positions are supervised by the national specialist advisory committee. The duration of specialist endodontics training is three years and usually examined under the broader subject of Restorative Dentistry. ${ }^{2}$ At the end of the specialist training, either a residency certificate is awarded or a master degree is conferred, depending upon where the training was done. However, the exit level qualification is the membership diploma in endodontics by the Royal College of Surgeons in Edinburgh, Glasgow or London. In Ireland, the exit level diploma is

Associate Professor, Operative Dentistry, Chief of Dental Services, Aga Khan University Karachi, Pakistan.

Corresponding author: “Dr. Farhan Raza Khan” < farhan.raza@aku.edu > the fellowship in endodontics awarded by the faculty of dentistry of the Royal College of Surgeons of Ireland.

In Australia and New Zealand, the model of endodontics training is quite similar to what is being followed in UK and Ireland. However, the exit level qualification is known as the fellowship in endodontics awarded by the Royal Australasian College of Dental Surgeons in Australia. ${ }^{3}$

In India, the modus operandi for all dental subjects specialist training is university based post-graduation (MDS). There are no CPSP or RCS type third party assessment; the Dental Council of India is the apex body for dentistry that regulates the profession as well as undergraduate/ post graduate curriculum and examinations. ${ }^{4}$ The subject is named as Conservative Dentistry \& Endodontics. MDS is the degree awarded after 3-4 years of specialist training in this subject.

In Pakistan, endodontics is the part of operative dentistry specialty. The mainstay of dental specialty training is the residency pathway prescribed by the College of Physicians and Surgeons of Pakistan (CPSP), commonly known as the FCPS training. Although, alternate specialty training pathway exists in the form of university post-graduation, also known as MDS training. The similarities and differences in the two pathways have been discussed in an earlier paper. ${ }^{5}$

Operative dentistry is a unique specialty; it's special because from this specialty, a number of other specialties and sub-specialties have emerged. Thus, it's justified to label it as the mother of other dental specialties. Another reason of the uniqueness of this specialty particularly in Pakistan, is its misnomeric nomenclature. In most part of the globe, the definition of operative dentistry has changed and has narrowed down to just "preparing teeth (enamel and dentine part only) for direct restorations, inlays, onlays and veneers and some procedures such as tooth bleaching and micro or 
macro-abrasions" and that's all about it. As per the most authentic textbook of operative dentistry, this specialty deals with the art and science of diseases and management of those defects of teeth that do not require full coverage restorations. ${ }^{6}$ Thus, the text book scope of operative dentistry per se, precludes the diseases of dental pulp and management of defects of teeth with extra coronal restorations. ${ }^{7,8}$

Interestingly, if the internationally accepted definition of operative dentistry is being followed, the ownership of dental pulp is totally missing in the name of this specialty in Pakistan. The correct name of the specialty in our context, should have been operative dentistry- endodontics. ${ }^{9}$ The clinicians of this discipline spent over $70 \%$ of their time in endodontics (managing pulp and peri-radicular diseases) similarly, over $70 \%$ of the curriculum of the specialty is endodontics. The assessment of competence of the trainees is also in accordance with that emphasis on endodontics. This has been discussed in length in a paper where Pakistani, Indian and British training and assessment models of this specialty were compared. ${ }^{10}$

An interesting thing about this specialty in Pakistan is that it has two models. The first model is a narrow-spectrum, focused training on operative dentistry-endodontics where residents confine themselves to fillings, root canals, bleaching, veneers and maximum upto single crowns. For full coverage crowns, both operative dentistry and prosthodontics overlap. The narrow spectrum model of training is mostly offered in the dental college settings. The strength of this model is that the graduates are the true specialists of the discipline who limit their practice to the perimeters defined by the specialty. The core curriculum of the specialty should enable them to make decisions about prevention and management of the diseases of the enamel, dentine, pulp and peri-radicular tissues. A specialist dentist trained in this discipline must be competent at applying knowledge to manage caries, tooth wear, hypoplastic defects of teeth and should be able offer predictable outcome in orthograde and retrograde endodontics.

The second model of training is a broad-spectrum, extended boundary residency where in addition to operative dentistry- endodontics, components of fixed prosthodontics and oral implantology have been inculcated in the residency curriculum. Obviously, these additions are not made at the cost of the primary specialty but rather as value added skills that might benefit the future clinicians in their future practice. This model of training is mainly offered at hospital-based residency programs (e.g. the Aga Khan University). At the outset, this model of training appears attractive as the training and skills set offered to the resident is above and beyond of what is being offered to their counterpart in sister institutions, however, there are certain issues and challenges in this model. Firstly, it defeats the purpose of specialty training as the idea of training the specialists is to train master clinicians for future generations who limit their practice to specific area so as to offer superior clinical outcomes. Secondly, when it comes to applying for any international qualifying examination at any of the royal colleges in UK and Ireland, presence of fixed prosthodontics and oral implantology in the log book would act as a contaminant in the fidelity of specialty training evidence. Lastly, having two genres of operative dentists- endodontists in the country is not a healthy trend where the former ones acting as true specialists and latter ones behaving as advanced general dentists.

In simpler terms, if these two models are extrapolated to general surgery training, the first genre of surgeons would be confined to carrying out surgery of appendix, gall bladder and liver whereas the second variety of surgeons would be doing every general surgery procedure including orthopedics and urology. Is there a way to manage the disparity existing between the two models of operative dentistry training? The answer is yes; the solution lies in the finding the middle ground. The senior teachers and examiners of this specialty should develop a consensus on limiting the curriculum of Implantology and fixed Prosthodontics in the core curriculum. It imperative to have following distribution in the syllabus and table of specification in the examination: endodontics $70 \%$, operative dentistry $20-25 \%$, oral implantology and fixed prosthodontics 5-10\%. Similarly, with the establishment of prosthodontics, periodontology, and pediatric dentistry as standalone specialties in the university hospitals, the natural evolution would take place and clinicians of those disciplines are likely to claim their territory back from the operative dentistry fellows. Thus, a harmony in the scope and domain of dental specialties will be amicably achieved.

\section{REFERENCES}

1. https://www.aae.org/specialty/education-events/academics/advancedprograms-in-endodontics/[accessed on 22nd Feb 2021].

2. https://www.rcseng.ac.uk/education-and-exams/exams/ search/membership-in-restorative-dentistry/[accessed on 22nd Feb 2021].

3. https://racds.org/education/specialist-dental-practice/fellowshipsdp/[accessed on 22nd Feb 2021].

4. https://collegedunia.com/courses/master-of-dental-surgery-mdsconservative-dentistry-and-endodontics [accessed on 22nd Feb 2021].

5. Khan FR. Dilemma of dental graduates seeking specialty training in Pakistan: MDS vs. FCPS. J Pak Dent Assoc. 2017; 26:44-5. https://doi.org/10.25301/JPDA.262.44 
6. Heymann H, Swift EJ, Ritter AV, Sturdevant CM. Sturdevant's Art and Science of Operative Dentistry. 2013. St. Louis, Mo: Elsevier/Mosby.

7. Mjor IA. Dentin and pulp: Endodontics or operative dentistry. J Dent Res. 1995;74:1535.

https://doi.org/10.1177/00220345950740090501

8. Mjor IA, Wilson NHF. What are you operative dentistry? Oper Dent.1996;21:1-3.
9. Khan FR, Khattak S, Ansari BB. Change in the specialty's name, from "Operative Dentistry" to "Endodontics \& Operative Dentistry". J Pak Dent Assoc. 2012;21:195-96.

10. Khan FR. Similarities and differences in specialty training of conservative dentistry and endodontics (India), operative dentistry (Pakistan) and restorative dentistry-endodontics (United Kingdom). J Pak Med Assoc. 2020;70:320-23.

https://doi.org/10.5455/JPMA.2934 